\title{
FRACTIONS: CONCEPTUAL AND DIDACTIC ASPECTS
}

\section{Sead Rešić ${ }^{1}$ \\ Ismet Botonjić \\ Maid Omerović}

Professional paper

Department of Mathematics, Faculty of Sciences, University of Tuzla, B\&H

Faculty of Education Travnik, B\&H

Received: 10.08 .2016

Accepted: 11.09.2016

\begin{abstract}
Fractions represent the manner of writing parts of whole numbers (integers). Rules for operations with fractions differ from rules for operations with integers. Students face difficulties in understanding fractions, especially operations with fractions. These difficulties are well known in didactics of Mathematics throughout the world and there is a lot of research regarding problems in learning about fractions. Methods for facilitating understanding fractions have been discovered, which are essentially related to visualizing operations with fractions.
\end{abstract}

Key words: fractions, numerator, denominator, manipulative and visual representation, proportional reasoning

\section{INTRODUCTION}

Fractions represent the manners of writing parts of integers. For example, if four children divide a chocolate among themselves equally, each child will have $1 / 4$, or in words, one quarter of the chocolate. If a child gets two quarters of the chocolate, he/she will have $2 / 4$, which is the same as one half of the chocolate. Therefore, $2 / 4=1 / 2$.

The process of teaching and learning about fractions is most certainly one of the most researched areas since the very beginnings of research in Mathematical education, possibly because this area (alongside the question of decimal numbers) presents one of the most obvious areas of failure in schools around the world (Fandiño Pinilla, 2007).

The origin of the fraction symbol $\frac{m}{n}$ has not been determined, but it is certain that Leonardo Fibonacci Pisano used it in his work Liber Abaci, published in 1202. Terms 'numerator' and 'denominator' also share undetermined origin, but it is known that they entered common use in language in Europe during the $15^{\text {th }}$ century. The difference between 'pure', 'apocryphal' and 'specious' fractions dates back to the $18^{\text {th }}$ century. Decimal numbers were presented in a work by Simone of Bruges, better known as Stevin (1548-1620), who did not use the decimal character (comma or point), but rather a completely different set of markers for decimal numbers (Fandiño Pinilla, 2007).

The fraction $\frac{a}{b}$ consists of numerator (a) and denominator (b) and remains equivalent when both numerator and denominator are multiplied or divided by the same number. The fraction is written in the simplest form unless numerator and denominator share a common factor.

${ }^{1}$ Correspondence to:

Sead Rešić, Department of Mathematics, Faculty of Sciences, University of Tuzla, B\&H

Šabana Zahirovića 10, Tuzla, B\&H

Phone: +38761101230

E-mail: sresic@hotmail.com 
Arithmetic operations with fractions are subject to rules which differ from those for operations with integers.

Rule for summation and detraction of fractions with same denominators: $\frac{a}{b} \pm \frac{c}{b}=\frac{a \pm c}{b}$

Rule for summation and detraction of fractions with different denominators: $\frac{a}{b} \pm \frac{c}{d}=\frac{a d}{b d} \pm \frac{b c}{b d}=\frac{a d \pm b c}{b d}$

Rule for multiplying fractions: $\frac{a}{b} \times \frac{c}{d}=\frac{a c}{b d}$

Rule for dividing fractions: $\frac{a}{b}: \frac{c}{d}=\frac{a}{b} \times \frac{d}{c}=\frac{a d}{b c}\left(=\frac{a / c}{b / d}\right)$ Understanding fractions and operations with fractions, as research and practice demonstrate, falls into the category of problematic segments of Mathematics teaching. That is why it is important that teachers be well trained and motivated to help students gain best possible knowledge in Mathematics and make that knowledge useful in further education, as well as in life and work.

\section{EARLY INTRODUCTION TO FRACTIONS}

Children understand the concept of division to equal parts. Children only four years of age are able to divide an array of objects equally among a small number of recipients (for example, divide twelve cookies among three people). At the age of five, children are able to divide one object among more recipients (for example, one candy stick). Beside this, children understand proportional relations very early. For example, a 6-year-old is able to establish equivalent proportions which represent various geometric figures or everyday objects (for example, $1 / 2$ of a pizza is a result of division, as is $1 / 2$ of a chocolate bar). This early knowledge can be employed to introduce the concept of fractions, through connection between the student's intuitive knowledge and formal concepts of fractions.

Teachers are advised to start with simple distribution activities, which include dividing a group of objects equally between a small group of recipients. Activities should include groups of objects which can be equally distributed among recipients, without leftovers (for example, distribute six cookies between two people). Teacher can describe the number of objects and recipients, so that student can determine how many objects each recipient gets. Students should be motivated to use concrete objects, drawings or other types of presentations which help them solve the problems. Teachers should emphasize that each recipient must get an equal number. As the students' knowledge improves, the number of objects to be divided, as well as recipients increases.

Moreover, students can start solving problems of division which include dividing object into smaller pieces. For example, if two people share one cookie, each person gets $1 / 2$ of the cookie. Instead of asking how many objects each person will get, the question becomes 'how big a part of the object will each person get?' Teachers may begin with division of one object to two or more recipients, and then move on to dividing more objects. The first question may include four people who share one apple, while the more advanced question could include division of two apples between four people. It is recommended that teachers begin with activities of division which enable students to use halving strategies (dividing objects in two halves, dividing those halves in two, etc.) before they move forward to division activities which demand that students divide something into thirds, fifths, etc. Teachers may also begin with introducing formal names of fractions (for example, one half, one third, one quarter) and let students label their drawings of those parts accordingly.

Activities of division may be employed to help students understand the actual size of the fraction. For example, by dividing an object among two, three, four or five people, students can see that increasing the number of recipients diminishes the size of the part each recipient gets. This idea can be connected to the concept of fractions, so that students learn that $1 / 4$ is smaller than $1 / 3$, which is smaller than $1 / 2$ (Fazio \& Siegler, 2011).

\section{FRACTIONS ARE NUMBERS}

An efficient way to ensure that students will understand that fractions are numbers with a certain value is to use the number line during class and explaining the subject matter. The number line can be used for all fractions, illustrating how every fraction corresponds with certain value.

Teachers can also use measurement activities to help students understand that fractions are numbers. Measuring objects allows students to learn that fractions enable more precise measurement than integers alone. Using various paper strips or cards for measuring different objects in the classroom is a very handy activity. Students begin with that measuring sample and start measuring objects. When the length of an object is not equal to the sum of samples, the teacher will begin using labels which represent $1 / 2,1 / 4,1 / 3$, or other parts of the entire sample (Fazio \& Siegler, 2011). 
MANIPULATIVE AND VISUAL REPRESENTATION OF FRACTIONS

Teachers should allow students to locate and compare fractions on the number line. Placing different fractions on the number line enables students to compare their sizes and see that some fractions are equivalent, such as $3 / 4$ and $6 / 8$. Teachers may begin with a number line with already marked fractions, for example, section of the number line from 0 to 1 has marked eighths. This additional step with the number line with correctly marked segments eliminates students' potential difficulties. Students should be asked to place both fractions marked on the number line (for example, 6/8) and fractions whose denominator is a common factor or multiple of an individual fraction marked on the number line (for example, $3 / 4$ and 12/16). It is also important to include fractions which are equivalent to integers (for example, $8 / 8$ ), so that students can understand that integers can be written as fractions.

In order to help students understand and compare fractions with different denominators, the number line can be marked with one unit of 1 above and the other unit of 1 below. For example, if the student is asked to compare $3 / 5$ and $7 / 8$, the teacher can divide the number line into fifths above the line and into eighths below the line. As students make progress, these additional supporting aids can be removed, do that students can use number lines with minimal markings, for example with marked end points and possibly the center point.

Number lines can also be used to expand students' concept of fractions by implementing negative fractions and fractions whose value is bigger than 1 ,

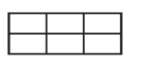

a)

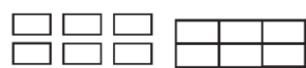

b) alongside decimal numbers and percentages. For example, students can be asked to place fractions such as $14 / 3$ on the number line with values from -1 on the left, to +1 on the right, with 0 in the middle. Similar strategy can be employed to help students connect the concepts of decimal numbers, fractions and percentages. Students can place various numbers, such as $3 / 5,0.25,33 \%$ on the same number line with 0 to 1 interval. In order to emphasize that fractions and decimal numbers can represent the same value, students can place fractions and their decimal equivalents on the same number line.

Finally, number lines can be useful in demonstrating the concept of fraction density. One of the ways to differentiate between fractions and integers is demonstrating the fact that there is an infinite number of fractions between any two fractions. This concept could be difficult for students to understand, but it can be illustrated using number lines. Students may begin with a number line which represents an integer (for example, 0 to 1) and split it into two halves. After that, students may keep halving the halves and make quarters, then eighths, sixteenths, and so on. This way, students can learn that every fraction can be divided into smaller fractions (Fazio \& Siegler, 2011).

Visual presentation of fractions helps developing conceptual understanding of calculating procedures.

For example, a bar of chocolate is usually dividend by lines into equal squares, which makes it easier to divide it into pieces. There can be a chocolate bar with marked division into 6 equal parts, so that every piece represents $1 / 6$, that is, one sixth of the entire chocolate. If the chocolate is divided among six people, everyone will get one piece. That piece is one sixth of the chocolate (Figure 1a)

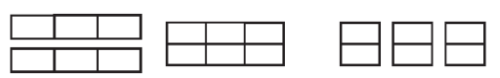

c)

Figure 1. Dividing into equal parts (from www.mathcentre.ac.uk)

If the chocolate is vertically divided into two parts, each part represents $1 / 2$ or $3 / 6$ of the chocolate (Figure $1 b)$. It is clearly demonstrated that that $1 / 2=3 / 6$. If the chocolate is horizontally divided in three parts (Figure 1 c) it can be seen that $1 / 3=2 / 6$.
Operations with mixed and improper fractions can be similarly illustrated. 

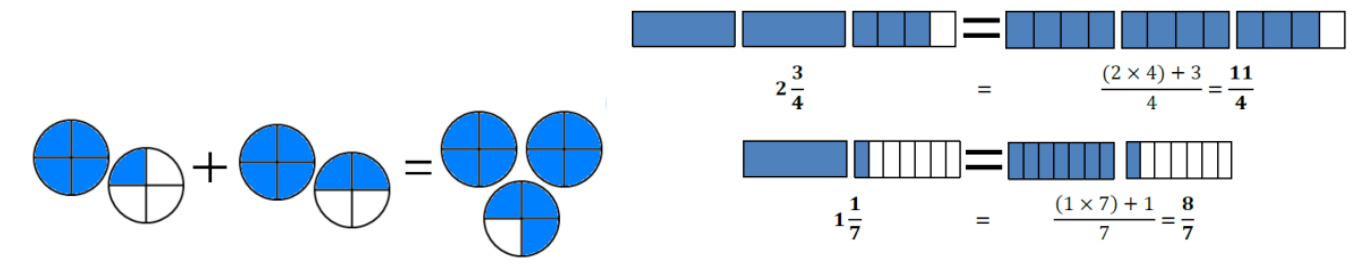

$\frac{5}{4}+\frac{6}{4}=\frac{5+6}{4}=\frac{11}{4} \quad \begin{gathered}\text { Figure 2. Illustration of operations with mixed and improper fractions (from } \\ \text { www.georgebrown.ca/tlc) }\end{gathered}$

One of the ways to improve conceptual understanding is employing manipulative and visual presentations of fractions. Studies which explored arythmetics of fractions using visual presentation of fractions have shown positive effects on students' abilities of calculation (Fazio \& Siegler, 2011).

Visual representation can be used to help with illustrating that common denominator is required in adding and subtracting fractions. For example, teacher can demonstrateaddition by using fractions of an object (i.e. $1 / 3$ of a rectangle and $1 / 2$ of a rectangle). Placing $1 / 3$ of the rectangle and $1 / 2$ of the rectangle together into a third rectangular can help the teacher show the approximate sum of the two. The teacher can then demonstrate that $1 / 3$ of the rectangle is the same as $2 / 6$, that $1 / 2$ is the same as $3 / 6$, and the sum is precisely $5 / 6$ of the rectangle. This type of concrete demonstration can help students understand why a common denominator is needed for adding and subtracting fractions.

Visual presentations can help students understand that multiplying fractions involves deriving fractions from other fractions. For example, in illustrating multiplication of fractions $1 / 4$ and $2 / 3$, students can start with a square, divide it with vertical lines for thirds and horizontal lines for fourths (quarters). Then, they can divide horizontally and vertically the two adjacent rectangles which together form a rectangle with sides of $1 / 4$ and $2 / 3$. The surface of this marked rectangle is $2 / 12$ of the initial square, and it equals the product of its sides which are $1 / 4$ and $2 / 3$. Therefore, the result is $1 / 4 \times 2 / 3=2 / 12$, which visually demonstrates the rule for multiplying fractions (See Figure 3 below).

1

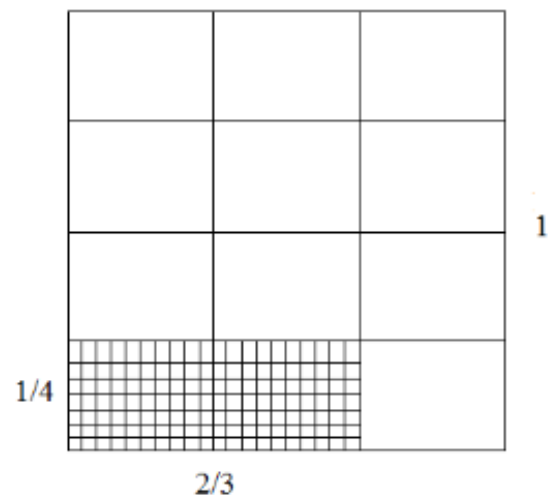

Figure 3. Visual demonstration for multiplying fractions

One of the ways to conceptualize division is to consider how many divisors are contained in the dividend. For example, $1 / 2: 1 / 4$ is the same as the question "How many quarters are there in one half?". Fraction strips can be used to illustrate division of fractions. For the above task, students can take two equally long strips, one of which is divided into two halves and the other one into four quarters. This way, students can discover how many quarters equal one half. This activity can also be done using number lines. Teacher should draw a number line with halves marked above the line and quarters marked below. Again, students can see that there are two quarters in one half (Fazio \& Siegler, 2011).

In order for students to understand fractions, they must see fractions in various constructions, including part of a whole, proportions and division. There are three categories of models for work with fractions: surface (i.e. $1 / 3$ of garden), length (i.e. $3 / 4$ of meter) and sets or quantities (i.e. $1 / 2$ of the classroom). 
Dividing into parts and repetition are good ways to help students understand the meaning of fractions, especially numerator and denominator. Dividing into equal parts is a way to build up on the knowledge of integers with the knowledge of fractions.

\section{ASSESSMENT BEFORE CALCULATION AND PROPORTIONAL REASONING}

Many students' mistakes with arithmetic of fractions could be avoided if students assess their answers before attempting to use the formal algorithm. However, it is not easy for many students to complete assessment of fractions. By practicing assessment, students improve their knowledge about the size of fractions as well as their understanding of arithmetic of fractions. Assessmentforces students to think about their answers and focus on what addition or multiplication of fractions really means, instead of merely following remembered rules without understanding.

When students solve problems in the area of arithmetic of fractions, they can be asked to assesstheir answerand explain their reasoning before calculating the answer. By assessingwhether their calculus answers were justified, students can recognize when they are using incorrect procedures or making mistakes applying correct procedures. For example, student can assess that $1 / 2+1 / 3$ must be bigger than $1 / 2$ and smaller than 1 , because $1 / 3$ is smaller than $1 / 2$ and $1 / 2+1 / 2=1$. If the student then incorrectly calculates that $1 / 2+1 / 3=$ $2 / 5$, teacher can emphasize that the answer cannot be correct, since $2 / 5$ is smaller than $1 / 2$. Teacher can then encourage the student to determine if the wrong answer was the result of incorrect application of procedure or using incorrect procedure altogether, and when the answer is the latter, student can be encouraged to complete or remember the correct procedure.

One of the strategies of assessment is using divisions. Divisions should be fractions which are well known to students, such as $0,1 / 2$ and 1 . Student can decide whether the given fraction between 0 and 1 is closer to zero, one half or one. For example, if the task is to add fractions $6 / 7$ and $5 / 8$, student can reasonably assume that $6 / 7$ is close to 1 and that $5 / 8$ is close to $1 / 2$, so the answer should be close to $1 \frac{1}{2}$.

Students can also consider the size of corresponding unit fractions (fractions where number 1 is the numerator) during assessment. If students understand that the size of the unit fraction reduces when the denominator increases (for example, $1 / 6$ is smaller $1 / 5$ ), this knowledge can help their assessment. For example, if the size of the sum of fractions $7 / 8+1 / 9$ should be assessed, students can reasonably assume that $7 / 8$ is smaller than 1 by $1 / 8$, and since $1 / 9$ is smaller than $1 / 8$, the answer will be that the sum is a bit smaller than 1 (Fazio \& Siegler, 2011).

Proportional reasoning is especially important for understanding percentages, proportions and ratios - the three interpretations of fractions which are not very often elaborated on as presentation about part/whole. Furthermore, proportional reasoning is often necessary in everyday life. Scaling a recipe up or down, calculating necessary groceries and determining the individual price of bought items in the shop, are activities from everyday real life which demand use of proportional reasoning.

It is important that students learn to solve problems within proportional reasoning using their own intuitive strategies before they learn the algorithmof crossmultiplication. Actually, even when students learn the algorithm, teachers should continue talking about students' informal reasoning strategies and how they result in the same answer as the algorithm. The reason for this is that students often do not know the meaning of the problem even after they learn the cross-multiplicationalgorithm, which leads to incorrect use of the algorithm. Teachers should emphasize that cross-multiplication algorithm makes it possible to find solutions for problems which would be difficult to solve using other strategies, but students should have conceptual basis about the term 'proportional reasoning', rather than only knowing how to solve problems using crossmultiplication.

\section{DIRECT COPING WITH USUAL LACK OF UN- DERSTANDING OF FRACTIONS IN ARITH- METICS}

Fractions are one of the most difficult areas for elementary school students. When adding or subtracting fractions, it is necessary to find the common denominator first, but it is not necessary when fractions are multiplied or divided. When we do find the common denominator, we add or subtract the nominators, not the denominators, contrary to the fact that when we multiply fractions, for example, we multiply both nominators and denominators (Siebert \& Gaskin, 2006).

Students around the world have difficulties learning about fractions. In many countries, the average students never gain conceptual knowledge about fractions. 
For example, on the national test, only $50 \%$ of American students in the eighth grade correctly align three fractions from smallest to biggest (National Council of Teachers of Mathematics, 2007). even in countries where students gain reasonably good conceptual understanding, such as Japan and China, fractions are considered a difficult subject. One of the reasons behind these difficulties is that fractions offer students their first lesson in learning that many characteristics which are correct for integers are incorrect for fractions. For example, with fractions, multiplication does not result in a bigger number than multiplied numbers individually, while division dooes not always lead to a number smaller that divided numbers individually, and numbers do not have only one successor. Overpowering the belief that characteristics that are correct for integers are also correct for all numbers is the main challenge. Even in secondary school, many students do not understand that there is an infinity of numbers between two fractions. Understanding fractions is of essential importance for learning algebra, geometry and other aspects of higher Mathematics (Fazio \& Siegler, 2011).

Students' difficulties with fractions often stem from lack of conceptual understanding. Many students see fractions as symbols with no meaning or they see nominator and denominator as separate numbers, instead of a whole. Students often confuse rules for arithemtics of integers with rules for arithmetics of fractions. It is important to identify the failure in understanding arithmetics of fractions and to directly point to the reaqson why this lack of understanding leads to incorrect answers, as well as why correct procedures lead to correct answers. Students will gain larger conceptual knowledge about arithmetics of fractions when they understand why procedures from arithmetics of integers do not apply to fractions, as opposed to when they merely learb new procedures for fractions.

Of all the well-known areas where lack of understanding is present, many students exhibit one or more of them. Three most common types of areas of lack of understanding will be discussed here, as well as what teachers can do to improve understanding in those areas:

Treating nominator and denominator as separate integers. When subtracting fractions, students often subtracts nominators and then denominators (for example, $5 / 8-1 / 4=4 / 4)$. These students make the mistake of treating nominator and denominator as separate integers, instead of treating the fraction as an individual number. Teachers can help students to overcome the lack of understanding by presenting clear and meaningful problems. For example, they can set a problem: "If you have $3 / 4$ of an orange and give $1 / 3$ to a friend, how much orange will you have left?" Students who perform the operation incorrect$1 y$, as described above, will answer " $2 / 1$ " or " 2 ". Asking students does it make sense to start with $3 / 4$ of an orange, give away one part, and have 2 oranges left, can helpstudents clearly see the problems with their lack of understanding. After presenting why their procedure was incorrect, students will find it more acceptable to learn the correct procedure.

Leaving denominator unchanged in problems with fraction multiplication. When fractions with same denominators are multiplied, students often leave the denominator unchanged (for example, $4 / 5 \times 1 / 5=$ 4/5). This mistake can occur because students work a lot more with adding fractions than multiplying them, which leads students to incorrectly apply the procedure for adding fractions where same denominators are used, to cases of fraction multiplication. Teacher can correct this mistake by explaining that the upper product actually means that it is $4 / 5$ of $1 / 5$. Since the problem requires a part of $1 / 5$, there cannot exist a number bigger that $1 / 5$ in the answer.

Lack of understanding of mixed numbers. Students often have difficulties solving problems with mixed numbers. Some students ignore the fraction part and focus only on the integer, (for example, $42 / 3-12 / 5=$ 3 ). Other students think that integers in the task must have the same denominator as fractions (for example, $3-\frac{2}{5}=\frac{3}{5} \frac{2}{5}=\frac{1}{5}$ ). There is also the mistake where the integer is multiplied by nominator of the fraction part (for example, $2 \frac{2}{5} \times \frac{5}{6}=\frac{4}{5} \times \frac{5}{6}=\frac{20}{30}$ ). All these mistakes maintain fundamental lack of understanding about what mixed numbers are and what is their size. Teachers should make sure to use proper, improper fractions and mixed numbers, while often transforming mixed numbers into improper fractions and vice versa (Fazio \& Siegler, 2011).

Successful teachers make mathematical connections and goals explicit in order to support students who are prone to focusing on issues of context in learning Mathematics (Anthony \& Walshaw, 2009). 


\section{CONTEXTS OF THE REAL WORLD}

Students can be good at creating real world context for problems presented in the form of fractions, and yet use calculus and arrive to incorrect answers when the problem is expressed in the form of fractions.For example, student can know that two halves make one whole, but still say that $\frac{1}{2}+\frac{1}{2}=\frac{2}{4}$. Teachers should emphasize and practice translating real world problems from the real world into fractions. This type of activity will help students connect their intuitive knowledge about fractions with formal symbols.

When presenting percentage problems, as well as fractions and proportions, teachers should use multiple contexts. The key goal is to achieve that students understand similarities between similar problems presented in different contexts. Teachers should help students identify key characteristics of problems. Problems should be presented using real life contexts which have meaning for students. For example, students can learn to compare prices looking for a oneunit price. If four candies can be bought with 3 marks, or six candies for 4.25 marks, which price is a better deal? (Fazio \& Siegler, 2011).

\section{TEACHER'S UNDERSTANDING}

Teachers should know how to explain why a certain algorithm is applicable. For example, almost all teachers know that the problem of dividing fractions can be solved through a procedure called "find the inverse number and multiply". However, many teachers lack deeper understanding of why this procedure is successful.

It is important for teachers to know not only concepts of fractions they learned at their education level, but also concepts which precede and follow. If the teacher understands what the student learned earlier, they can build on what the student already knows and can better identify the source of students' lack of understanding. Knowledge of materials students will learn later helps teachers understand what students should learn during the academic year in order to gain solid basis for later education.

Deeper understanding of concepts of fractions is also necessary for efficient use of visual presentations in class. During professional development, teachers need to learn to use presentations efficiently and connect them with concepts taught in class. Various presentations are more or less appropriate for various situations. For example, diagrams may be useful in explaining the scenario of division (for example, dividing three cakes in five equal parts) and understanding division of fractions. Number lines can help students focus on the size of the fractions, while surface models (rectangles or circles which are partially shaded) can illustrate fraction presentation about part/whole. Teachers should also be familiar with difficulties which may occur while using visual presentations. For example, students can have problems with drawing equal parts, or can wrongly interpret the length of the number line, placing $1 / 2$ in the middle of a number line with division markers from 0 to 5 , instead of placing it near 0 .

Through activities in professional development, teachers should learn how students develop understanding of concepts of fractions and understand difficulties students face in correct understanding of fractions. One of the ways for teachers to gain deeper understanding of how students learn to present fractions is to examine students' written assignments and video recordings of their process of solving problems with fractions. Teachers should discuss among themselves the reasons why students have difficulties with a certain kind of problems, considering different points in students' knowledge about fractions. Teachers should also discuss about types of mistakes students usually make and lack of understanding in connected to each mistake. This kind of discussion can also enable teachers to learn about types of problems related to fractions which they can ask students to solve, in order to diagnose the source of students' lack of understanding. When teachers understand why their students have difficulties, they can pay more attention to appropriate problems in understanding fractions in the classroom (Fazio \& Siegler, 2011).

\section{CONCLUSION}

Fractions are an important springboard for learning advanced Mathematics. They are also often used in everyday life. Still, many students are struggling with fractions, even after years of education. We believe that recommendations presented in this paper will help empower teaching fractions, and increase the number of students who understand fractions and correctly solve problems in arithmetic of fractions. It is important to emphasize that fractions are a difficult area of study. Even after implementing recommendations from this paper, students will not immediately understand what fractions are and how they are different from integers. However, frequent use of ideas and various examples, as well as above mentioned recommendations, students' understanding of fractions will improve. 
Recommendations are designed to be implemented as a whole, even though each recommendation on its own should contribute to conceptual understanding. Students need deep conceptual understanding of fractions in order to learn them efficiently and be able to remember what they learned. When students have superficial understanding of fractions, the mere fraction symbol is meaningless, as is the arithmetic procedure used for fractions, which looks arbitrary and easy to confuse. Cultivating conceptual understanding enables teachers to help students understand that fractions are real numbers and that arithmetic of fractions is a meaningful procedure, and not only a string of arbitrary steps. Conceptual understanding is difficult to gain, but it is of vital importance for ensuring deep and permanent understanding of fractions and their arithmetic.

\section{REFERENCES}

Fandiño Pinilla, M.I. (2007). Fractions: conceptual and didactic aspects. Acta Didactica Universitatis Comenianae. 7, 23-45.

Fazio, L. \& Siegler, R. (2011). Teaching fractions. Educational practices series - 22. International Academy of Education \& International Bureau of Education.

National Council of Teachers of Mathematics. (2007). The learning of mathematics: 69th NCTM yearbook. Reston, VA: National Council of Teachers of Mathematics.

Siebert, D. \& Gaskin, N. (2006). Creating, Naming, and Justifying Fractions. Teaching Children Mathematics, April 2006. 